\title{
A Fuzzy Approach to Risk Based Decision Making
}

\author{
Omar Khadeer Hussain ${ }^{1}$, Elizabeth Chang ${ }^{1}$, Farookh Khadeer Hussain ${ }^{1}$, and \\ Tharam S. Dillon ${ }^{2}$ \\ ${ }^{1}$ School of Information Systems, Curtin University of Technology, Perth, Australia \\ \{Omar.Hussain, Elizabeth.Chang, Farookh.Hussain\}@cbs.curtin.edu.au \\ ${ }^{2}$ Faculty of Information Technology, University of Technology, Sydney, Australia \\ tharam@it.uts.edu.au
}

\begin{abstract}
Decision making is a tough process. It involves dealing with a lot of uncertainty and projecting what the final outcome might be. Depending on the projection of the uncertain outcome, a decision has to be made. In a peer-topeer financial interaction the trusting agent, in order to analyse the Risk, has to consider the possible likelihood of failure of the interaction and the possible consequences of failure to its resources involved in the interaction before concluding whether to interact with the probable trusted agent or not. Further, it may also have to choose and decide on an agent to interact with from a set of probable trusted agents. In this paper, we propose a fuzzy risk based decision making system that would assist the trusting agent to ease its decision making process.
\end{abstract}

\section{Introduction}

The trusting agent can make an informed decision of whether to interact with a probable trusted agent or not, by analysing before-hand the possible level of risk that could be present in interacting with it. The Australian and New Zealand Standard on Risk Management, AS/NZS 4360:2004 states that risk identification is the heart of risk management [1]. Hence, risk should be identified in order to analyse and manage it better. Risk is important in the study of behaviour in e-commerce because there is a whole body of literature based in rational economics that argues that the decision to buy is based on risk-adjusted cost-benefit analysis [11]. Thus, it commands a central role in any discussion of e-commerce that is related to a transaction.

The definitions of risk in literature highlight and emphasise the possible loss in an interaction. To mention some such definitions; March et al. defines risk more by the magnitude of the value of the outcome rather than by its likelihood [2]. Luhmann defines risk in a transaction as where the possible damage might be more than the advantage sought [3]. Mayer et al. conclude that risk is present in the transaction only if the negative outcome outweighs the positive outcome [4]. In contrast to this definition, Rousseau et al. measure risk as the potential negative consequence and probability of failure [5]. Sztompka defines risk as the probability of the loss of the resources invested [6]. Grazoli et al. view risk as the consumers' perception of 
uncertainty and adverse consequences of engaging in an activity [7]. Cheung et al. define risk as having two dimensions; one related to the uncertainty or probability of loss notion and the other related to a consequence of the importance of the notion of loss [8]. Hussain et al. [9] define risk in the context of peer-to-peer business interactions as the likelihood that the trusted agent might not act as expected according to the trusting agent's expectations in a given context and at a particular time once the interaction begins, resulting in financial loss of the resources involved in the interaction. Risk is seen as a combination of:

a) The possibility of failure in achieving the outcome; and

b) The cost of the outcome when it occurs, usually the loss incurred, which is related to Risk.

Risk evaluation involves the trusting agent having to determine before-hand the possibility of failure of the interaction and the subsequent possible consequences for its resources involved in the interaction. This is different from trust evaluation. Trust evaluation measures the belief that the trusting agent has in a probable trusted agent in attaining its desired outcomes if it interacts with the probable trusted agent.

In a decentralised interaction carried out in service oriented architecture, the trusting agent should first analyse the possible level of risk that could be involved in dealing with a probable trusted agent. Doing so would help the trusting agent to decide whether to interact with the probable trusted agent or not, or to choose an agent to interact with, from a set of probable trusted agents. But decision making is a tough process. It involves dealing with a lot of uncertainty and projecting what the outcome might be. Depending on the projection of the final outcome a decision has to be made. So, in order to ease the decision making process for the trusting agent, or to further strengthen its previous belief of proceeding in an interaction with a probable trusted agent or not, we propose the utilisation of a fuzzy system in decision making. The trusting agent can ease its decision making process by utilising a fuzzy approach to risk based decision making. The proposed fuzzy model combines the possibility of failure of the interaction and the possible consequences of failure in interacting with a probable trusted agent and gives an output to the trusting agent. Fuzzy logic is used to model uncertainty [], and similarly, the decision making process deals with predicting the possible uncertain outcome and deciding the future course of action based on the predicted uncertain outcome.

In this paper, we propose and define a fuzzy risk based decision making system. The trusting agent inputs the aspects of risk related to the probable trusted agent to the fuzzy system and based on that it gets an output whether to proceed or not in the interaction with the probable trusted agent. The proposed system is explained in the next sections. This paper is organised into 7 sections. In Section 2 we discuss our previous work of determining the different constituents for analysing the possible risk in an interaction. From section 3 to section 6 we propose and demonstrate the fuzzy risk based decision making system and finally in section 7 we conclude the paper. 


\section{Analysing the Possible Risk in an Interaction}

As discussed earlier, the trusting agent can make an informed decision whether to interact or not with the probable trusted agent by analysing the possible risk that could be present. Risk analysis by the trusting agent in its future interaction with the probable trusted agent can be done by:

- Determining the possibility of failure of the interaction; and

- Determining the possible consequences of failure of the interaction.

Hence, the trusting agent should consider these two factors for each probable agent in order to determine the possible risk associated in interacting with each agent. Based on the analysis, the trusting agent can make an informed decision of whether to interact or not with a particular trusted agent.

\subsection{Determining the Possibility of Failure of the Interaction}

The possibility of failure of an interaction is the extent to which the trusting agent thinks that it might not achieve its desired outcomes in interacting with a probable trusted agent. The trusting agent can determine the possibility of failure in interacting with a probable trusted agent by analysing its capacity for not completing the interaction according to how it wants. Further, the trusting agent should analyse the possibility of failure in interacting with a probable trusted agent according to the context and criteria of its future interaction with it. The trusting agent's interaction with the probable trusted agent is in the future state of time. It has to analyse the possibility of failure in interacting with a probable trusted agent in two stages. They are:

1. Pre-interaction start time phase

2. Post-interaction start time phase

Pre-interaction start time phase refers to the period of time before the trusting agent starts its interaction with the probable trusted agent, whereas post-interaction start time phase is the period of time after the trusting agent starts its interaction with the probable trusted agent. The possible interaction of the trusting agent with a probable trusted agent will be in the future state of time i.e. in the post-interaction start time phase and, hence, the possibility of failure of the interaction must be determined in that phase. In order for the trusting agent to determine the possibility of failure of a probable trusted agent in the post-interaction start time phase, it should know its possibility of failure according to the specific context and criteria as that of its future interaction until the pre-interaction start time phase. Based on the values achieved for the probable trusted agent until the pre-interaction start time phase, the trusting agent can determine the possibility of failure in the post-interaction start time phase.

In order to determine the possibility of failure in interacting with a probable trusted agent we defined the term 'Riskiness' in Hussain et al. []. Riskiness is a numeric value that is assigned to the trusted agent from the Riskiness scale. The Riskiness value shows the level of possibility of failure on the Riskiness scale. The Riskiness scale (as shown in Figure 1) ranges from 0 to 5 , with the value 0 representing the highest level of possibility of failure and the value 5 denoting the lowest level of possibility of failure. The Riskiness value for a probable trusted agent 
in an interaction is assigned to it after determining its capacity to complete the interaction according to the expectations of the trusting agent. Each Riskiness value on the Riskiness scale has a corresponding level of possibility of failure associated with it.

\begin{tabular}{|c|c|c|c|c|}
\hline $\begin{array}{c}\text { Riskiness } \\
\text { Levels }\end{array}$ & $\begin{array}{c}\text { Magnitude of } \\
\text { Failure }\end{array}$ & $\begin{array}{c}\text { Riskiness } \\
\text { Value }\end{array}$ & $\begin{array}{l}\text { Possibility of } \\
\text { Failure Levels }\end{array}$ & Star Rating \\
\hline Unknown Risk & - & $\therefore$ & Unknown & Not Displayed \\
\hline Totally Risky & $\begin{array}{c}91-100 \% \\
\text { Possibility of Failure }\end{array}$ & 0 & $\begin{array}{l}\text { Definite Total } \\
\text { Failure }\end{array}$ & Not Displayed \\
\hline $\begin{array}{l}\text { Extremely } \\
\text { Risky }\end{array}$ & $\begin{array}{c}71-90 \% \\
\text { Possibility of Failure }\end{array}$ & . & Extremely High & From 此 to \\
\hline Largely Risky & $\begin{array}{c}51-70 \% \\
\text { Possibility of Failure }\end{array}$ & 2 & Largely High & From 致你 to \\
\hline Risky & $\begin{array}{c}26-50 \% \\
\text { Possibility of Failure }\end{array}$ & 3 & High & 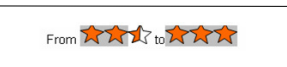 \\
\hline $\begin{array}{l}\text { Largely } \\
\text { UnRisky }\end{array}$ & $\begin{array}{c}11-25 \% \text { Possibility of } \\
\text { Failure }\end{array}$ & 4 & $\begin{array}{c}\text { Significantly } \\
\text { Low }\end{array}$ & From \\
\hline UnRisky & $\begin{array}{c}C-1 C \% \text { Possibility } \\
\text { of Failure }\end{array}$ & 5 & $\begin{array}{c}\text { Extremely } \\
\text { Low }\end{array}$ & From \\
\hline
\end{tabular}

Fig 1. Riskiness scale

The possibility of failure in interacting with a probable trusted agent in preinteraction time phase can be determined by the trusting agent by considering the past interaction history or soliciting recommendations from other agents. As mentioned by Carter and Ghorbani [10], reputation of a peer can be relied upon in case of total ignorance.

We have developed a methodology by which the trusting agent determines the possibility of failure in interacting with a probable trusted agent and assigns it with a Riskiness value in both the pre- and post-interaction start time phase in Hussain et al. [9]. Due to space limitation, we will not be discussing it here.

\subsection{Determining the Possible Consequences of Failure in an Interaction}

Another aspect of risk is the possible loss that could be incurred in an interaction. The trusting agent, in order to measure the possible risk in an interaction, should also determine the possible consequences of failure to its resources in interacting with the probable trusted agent apart from determining the possibility of failure in interacting with that particular probable trusted agent. In a peer-to-peer financial interaction, the possible loss that could occur to the trusting agent is usually the financial loss in its resources that are involved in the interaction.

The consequences of failure of an interaction will be modelled over a scale of 0 $100 \%$ representing the loss incurred. The possible consequences of failure in an interaction are determined only in the post-interaction start time phase, as this is the period in which the trusting agent interacts with the probable trusted agent and its resources are at stake. We have developed a methodology in Hussain et al. [9] through which the trusting agent can determine the possible consequences to its resources involved in the interaction. 
Once the trusting agent determines the possibility of failure and the possible consequences of failure of an interaction, it should combine those to determine the possible risk in order to assist decision making. As mentioned earlier, decision making is a tough process. It involves dealing with a lot of uncertainty. The trusting agent, in spite of determining the possibility of failure and the possible loss of resources, might still be uncertain or undecided whether to interact with the particular trusted agent. To alleviate this problem, we propose the utilisation of a fuzzy system which will help the trusting agent in its decision making process. We will propose the fuzzy system in the next section.

\section{Developing a Fuzzy Risk Based Decision Making System}

Once the possibility and consequences of failure have been determined, we need a systematic approach to synthesise these two constituents of risk into a given Risk Value for making an informed decision. We propose the use of a fuzzy approach to enable this. The main aim of the fuzzy decision making system is to assist the trusting agent with the decision making process. In order to achieve that, we propose that the trusting agent inputs the relative values of the probable trusted agent to the fuzzy system, which in turn evaluates them according to the pre-defined rules. Based on the evaluation of the rules, an output is given to the trusting agent. The output of the fuzzy system will be in the form of 'Proceed (x)' or 'Don't Proceed (x)', where the value ' $x$ ' quantifies the strength to which the output qualifies.

The input from the universe of discourse (UoD) to the fuzzy system must be in fuzzy variables. Fuzzy variables are linguistic objects rather than numbers. Hence, the inputs (namely Pre-interaction: Possibility of failure, Post-interaction: Possibility of failure and Consequences) of the probable trusted agent must be in linguistic terms to the fuzzy system. In order to do this, each of the inputs uses the following fuzzy sets to space their inputs values.

\section{\{Low, Medium and High $\}$}

Further in this paper, we will represent the inputs to the fuzzy system by their acronyms.

- Pre-interaction: Possibility of failure is represented as Pre-I: PoF

- Post-interaction: Possibility of failure is represented as Post-I: PoF and

- Consequences as Consequences.

\section{Defining Rules for the Fuzzy Logic System}

According to the Mamdani approach [], in order for the fuzzy system to conclude at an output, it needs some rules to process the inputs. Linguistic rules in the fuzzy system consists of two parts, an antecedent (between the IF and THEN) and 
consequent (following THEN). There are 3 inputs to our fuzzy system and there are 3 fuzzy sets. Hence, the total number of rules is: $3^{3}=27$. The rules are:

1. If Pre-I:PoF $=\mathbf{L}$ and Post-I:PoF $=\mathbf{L}$ and Consequences $=\mathbf{L}$, then Output $=\mathbf{P}$

2. If Pre-I:PoF $=\mathbf{L}$ and Post-I:PoF $=\mathbf{M}$, and Consequences $=\mathbf{L}$, then Output $=\mathbf{P}$

3. If Pre-I:PoF $=\mathbf{L}$ and Post-I:PoF $=\mathbf{H}$, and Consequences $=\mathbf{L}$, then Output $=\mathbf{D P}$

4. If Pre-I:PoF $=\mathbf{M}$ and Post- $\mathrm{I}: \mathrm{PoF}=\mathbf{M}$, and Consequences $=\mathbf{L}$, then Output $=\mathbf{P}$

5. If Pre-I:PoF= $\mathbf{M}$ and Post-I:PoF $=\mathbf{L}$, and Consequences $=\mathbf{L}$, then Output $=\mathbf{P}$

6. If Pre-I:PoF $=\mathbf{M}$ and $\mathrm{Post}-\mathrm{I}: \mathrm{PoF}=\mathbf{H}$, and Consequences $=\mathbf{L}$, then Output $=\mathbf{D P}$

7. If Pre-I:PoF $=\mathbf{H}$ and Post-I:PoF $=\mathbf{L}$, and Consequences $=\mathbf{L}$, then Output $=\mathbf{D P}$

8. If Pre-I:PoF $=\mathbf{H}$ and $\mathrm{Post}-\mathrm{I}: \mathrm{PoF}=\mathbf{H}$, and Consequences $=\mathbf{L}$, then Output $=\mathbf{D P}$

9. If Pre-I:PoF $=\mathbf{H}$ and Post-I:PoF $=\mathbf{M}$, and Consequences $=\mathbf{L}$, then Output $=\mathbf{D P}$

10. If Pre-I:PoF $=\mathbf{L}$ and Post-I:PoF $=\mathbf{L}$ and Consequences $=\mathbf{H}$, then Output $=\mathbf{D P}$ 11. If Pre-I:PoF $=\mathbf{L}$ and Post-I:PoF $=\mathbf{M}$, and Consequences $=\mathbf{H}$, then Output $=\mathbf{D P}$

12. If Pre-I:PoF $=\mathbf{L}$ and Post-I:PoF $=\mathbf{H}$, and Consequences $=\mathbf{H}$, then Output $=\mathbf{D P}$

13. If Pre-I:PoF $=\mathbf{M}$ and Post- $\mathrm{I}: \mathrm{PoF}=\mathbf{M}$, and Consequences $=\mathbf{H}$, then Output $=\mathbf{D P}$

14. If Pre-I:PoF $=\mathbf{M}$ and Post-I:PoF $=\mathbf{L}$, and Consequences $=\mathbf{H}$, then Output $=\mathbf{D P}$

15. If Pre-I:PoF $=\mathbf{M}$ and Post-I:PoF $=\mathbf{H}$, and Consequences $=\mathbf{H}$, then Output $=\mathbf{D P}$

16. If Pre-I:PoF $=\mathbf{H}$ and Post-I:PoF $=\mathbf{L}$, and Consequences $=\mathbf{H}$, then Output $=\mathbf{D P}$

17. If Pre-I:PoF $=\mathbf{H}$ and Post-I:PoF $=\mathbf{H}$, and Consequences $=\mathbf{H}$, then Output $=\mathbf{D P}$

18. If Pre-I:PoF $=\mathbf{H}$ and Post-I:PoF $=\mathbf{M}$, and Consequences $=\mathbf{H}$, then Output $=\mathbf{D P}$

19. If Pre-I:PoF $=\mathbf{L}$ and Post-I:PoF $=\mathbf{L}$ and Consequences $=\mathbf{M}$, then Output $=\mathbf{P}$

20. If Pre-I:PoF $=\mathbf{L}$ and Post-I:PoF $=\mathbf{M}$, and Consequences $=\mathbf{M}$, then Output $=\mathbf{P}$

21. If Pre-I:PoF $=\mathbf{L}$ and Post-I:PoF $=\mathbf{H}$, and Consequences $=\mathbf{M}$, then Output $=\mathbf{D P}$

22. If Pre-I:PoF $=\mathbf{M}$ and Post-I:PoF $=\mathbf{M}$, and Consequences $=\mathbf{M}$, then Output $=\mathbf{D P}$

23. If Pre-I:PoF $=\mathbf{M}$ and Post- $\mathrm{I}: \mathrm{PoF}=\mathbf{L}$, and Consequences $=\mathbf{M}$, then Output $=\mathbf{P}$

24. If Pre-I:PoF $=\mathbf{M}$ and Post-I:PoF $=\mathbf{H}$, and Consequences $=\mathbf{M}$, then Output $=\mathbf{D P}$

25. If Pre-I:PoF $=\mathbf{H}$ and Post-I:PoF $=\mathbf{L}$, and Consequences $=\mathbf{M}$, then Output $=\mathbf{D P}$

26. If Pre-I:PoF $=\mathbf{H}$ and Post-I: $\mathrm{PoF}=\mathbf{H}$, and Consequences $=\mathbf{M}$, then Output $=\mathbf{D P}$

27. If Pre-I:PoF $=\mathbf{H}$ and Post-I:PoF $=\mathbf{M}$ and Consequences $=\mathbf{M}$, then Output $=\mathbf{D P}$

\section{$5 \quad$ Membership Functions}

Membership functions are a graphical representation, represented by different shapes in order to determine the magnitude of participation of each input. No matter what shape any input or output is represented, the height or magnitude of the shape is usually normalised to 1 . The width of the shape represents the base of the function. In our fuzzy system, the range of two inputs i.e. Pre-I: PoF and Post-I: PoF is within $[0,5]$ and that of consequence is within [0-100\%]. We represent the gbell shape to determine the strength of the inputs and the triangle shape to determine the strength of the output in our system. The membership functions for the inputs and outputs to the fuzzy system are shown in the figures below. 


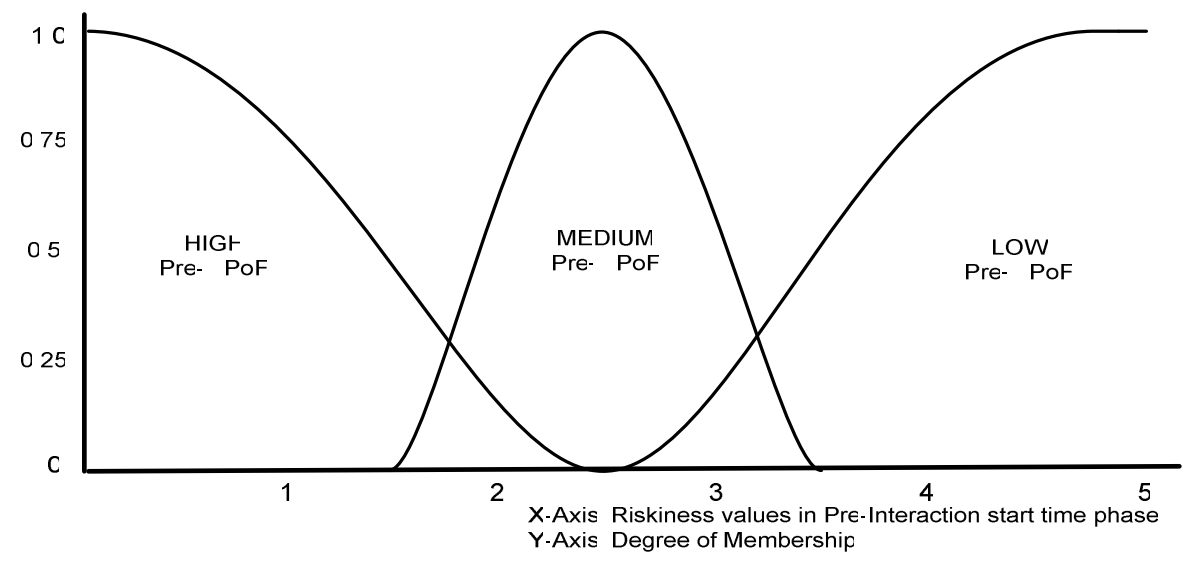

Fig 2. Membership function for the input "Pre-I: PoF"

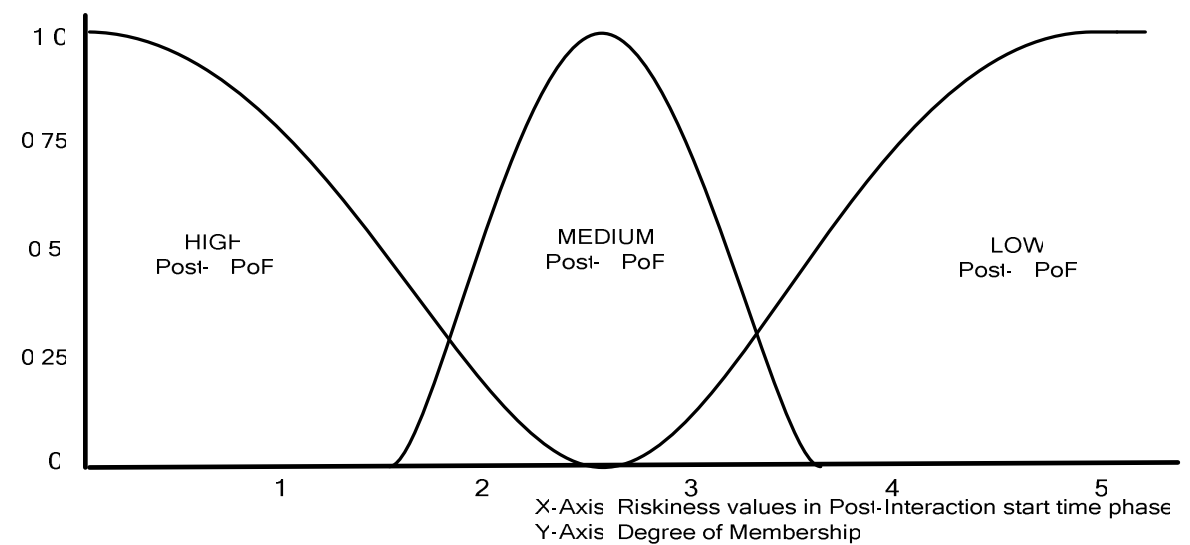

Fig 3. Membership function for the input "Post-I: PoF"

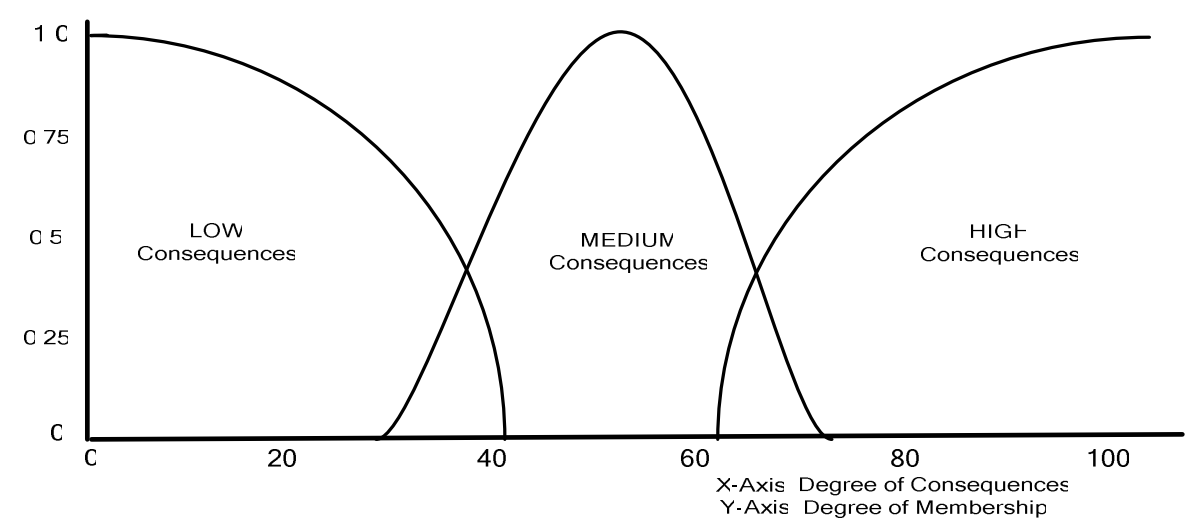

Fig 4. Membership function for the input "Consequences" 


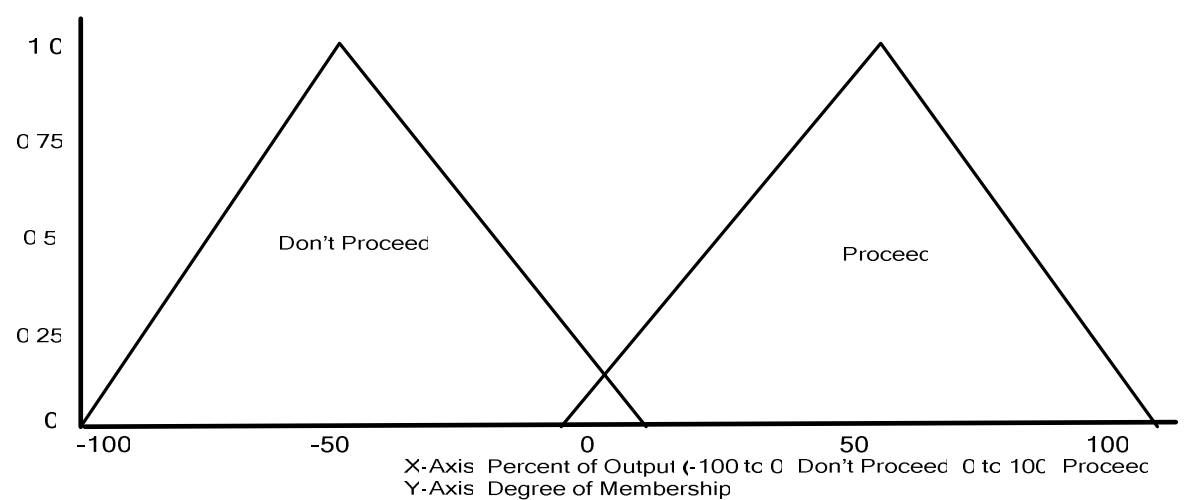

Fig 5. Membership function for the output "Proceed" or "Don't Proceed"

\section{Example of Fuzzy Risk Based Decision Making}

In order to understand better, let us suppose that a trusting agent utilises the fuzzy logic system in order to arrive at a conclusion whether to proceed or not in an interaction with a probable trusted agent. The input that it gives to the fuzzy logic engine is:

Pre-I: PoF: 3

Post-I: PoF: 2

Consequences of Failure of the Interaction: $\mathbf{5 2 . 9 0 \%}$

Plotting the inputs on their respective membership function gives the membership values for each input.

Pre-I: PoF, Low $\mu=0.16$ and Medium $\mu=0.62$

Post-I: PoF, Medium $\mu=0.62$ and High $\mu=0.16$

Consequences, Medium $\mu=\mathbf{0 . 9 8}$

Utilising the rule base, Table 2 gives the output for each rule.

Table 2

\begin{tabular}{|c|c|c|c|c|c|c|c|c|}
\hline & & Pre-I:PoF & & Post-I:PoF & & $\begin{array}{l}\text { Consequ } \\
\text { ences }\end{array}$ & & Output \\
\hline Rule 1 & If & 0.16 & $\&$ & 0.0 & $\&$ & 0.0 & then & $\begin{array}{l}\text { Proceed } 0.50 \& 0 \& \\
0=(0)\end{array}$ \\
\hline Rule 2 & If & 0.16 & $\&$ & 0.62 & $\&$ & $\mathbf{0 . 0}$ & then & Proceed $(0)$ \\
\hline Rule 3 & If & 0.16 & $\&$ & 0.16 & $\&$ & $\mathbf{0 . 0}$ & then & Don't Proceed (0) \\
\hline Rule 4 & If & 0.62 & $\&$ & 0.62 & $\&$ & $\mathbf{0 . 0}$ & then & Proceed (0) \\
\hline Rule 5 & If & 0.62 & $\&$ & 0.0 & $\&$ & $\mathbf{0 . 0}$ & then & Proceed (0) \\
\hline Rule 6 & If & 0.62 & $\&$ & 0.0 & $\&$ & 0.0 & then & Don't Proceed (0) \\
\hline Rule 7 & If & 0.0 & $\&$ & $\mathbf{0 . 0}$ & $\&$ & 0.0 & then & Don't Proceed (0) \\
\hline Rule 8 & If & $\mathbf{0 . 0}$ & $\&$ & 0.16 & $\&$ & 0.0 & then & Don't Proceed (0) \\
\hline Rule 9 & If & 0.0 & $\&$ & 0.62 & $\&$ & $\mathbf{0 . 0}$ & then & Don't Proceed (0) \\
\hline Rule 10 & If & 0.16 & $\&$ & 0.0 & $\&$ & $\mathbf{0 . 0}$ & then & Don't Proceed (0) \\
\hline
\end{tabular}




\begin{tabular}{|l|l|l|l|l|l|l|l|l|}
\hline Rule 11 & If & $\mathbf{0 . 1 6}$ & $\&$ & $\mathbf{0 . 6 2}$ & $\&$ & $\mathbf{0 . 0}$ & then & Don't Proceed (0) \\
\hline Rule 12 & If & $\mathbf{0 . 1 6}$ & $\&$ & $\mathbf{0 . 1 6}$ & $\&$ & $\mathbf{0 . 0}$ & then & Don't Proceed (0) \\
\hline Rule 13 & If & $\mathbf{0 . 6 2}$ & $\&$ & $\mathbf{0 . 6 2}$ & $\&$ & $\mathbf{0 . 0}$ & then & Don't Proceed (0) \\
\hline Rule 14 & If & $\mathbf{0 . 6 2}$ & $\&$ & $\mathbf{0 . 0}$ & $\&$ & $\mathbf{0 . 0}$ & then & Don't Proceed (0) \\
\hline Rule 15 & If & $\mathbf{0 . 6 2}$ & $\&$ & $\mathbf{0 . 1 6}$ & $\&$ & $\mathbf{0 . 0}$ & then & Don't Proceed (0) \\
\hline Rule 16 & If & $\mathbf{0 . 0}$ & $\&$ & $\mathbf{0 . 0}$ & $\&$ & $\mathbf{0 . 0}$ & then & Don't Proceed (0) \\
\hline Rule 17 & If & $\mathbf{0 . 0}$ & $\&$ & $\mathbf{0 . 1 6}$ & $\&$ & $\mathbf{0 . 0}$ & then & Don't Proceed (0) \\
\hline Rule 18 & If & $\mathbf{0 . 0}$ & $\&$ & $\mathbf{0 . 6 2}$ & $\&$ & $\mathbf{0 . 0}$ & then & Don't Proceed (0) \\
\hline Rule 19 & If & $\mathbf{0 . 1 6}$ & $\&$ & $\mathbf{0 . 0}$ & $\&$ & $\mathbf{0 . 9 8}$ & then & Proceed (0) \\
\hline Rule 20 & If & $\mathbf{0 . 1 6}$ & $\&$ & $\mathbf{0 . 6 2}$ & $\&$ & $\mathbf{0 . 9 8}$ & then & Proceed (0.16) \\
\hline Rule 21 & If & $\mathbf{0 . 1 6}$ & $\&$ & $\mathbf{0 . 6 2}$ & $\&$ & $\mathbf{0 . 9 8}$ & then & $\begin{array}{l}\text { Don't } \\
\text { (0.16) }\end{array}$ \\
\hline Rule 22 & If & $\mathbf{0 . 6 2}$ & $\&$ & $\mathbf{0 . 6 2}$ & $\&$ & $\mathbf{0 . 9 8}$ & then & $\begin{array}{l}\text { Don't } \\
\text { (0.62) }\end{array}$ \\
\hline Rule 23 & If & $\mathbf{0 . 6 2}$ & $\&$ & $\mathbf{0 . 0}$ & $\&$ & $\mathbf{0 . 9 8}$ & then & Proceed \\
\hline Rule 24 & If & $\mathbf{0 . 6 2}$ & $\&$ & $\mathbf{0 . 1 6}$ & $\&$ & $\mathbf{0 . 9 8}$ & then & $\begin{array}{l}\text { Don't } \\
\text { (0.16) }\end{array}$ \\
\hline Rule 25 & If & $\mathbf{0 . 0}$ & $\&$ & $\mathbf{0 . 0}$ & $\&$ & $\mathbf{0 . 9 8}$ & then & Pon't Proceed $(\mathbf{0})$ \\
\hline Rule 26 & If & $\mathbf{0 . 0}$ & $\&$ & $\mathbf{0 . 1 6}$ & $\&$ & $\mathbf{0 . 9 8}$ & then & Don't Proceed (0) \\
\hline Rule 27 & If & $\mathbf{0 . 0}$ & $\&$ & $\mathbf{0 . 6 2}$ & $\&$ & $\mathbf{0 . 9 8}$ & then & Don't Proceed (0) \\
\hline
\end{tabular}

The logical sum of the rules must be inferred first according to the possible output memberships before being defuzzified to produce a crisp output. We infer the logical sum of the rules by the Root-Sum-Square (RSS) method. The RSS method combines the effect of all applicable rules, scales the functions at their respective magnitude and computes the fuzzy centroid of the composite area. Compared with the other methods of inferring, the RSS gives the best weighted influence to all firing rules. Hence, it was chosen in this example as it includes all contributing rules. Other methods of defuzzification can also be utilised to defuzzify the logical sums of the rules.

For the ongoing example, an input of 3,2 and 52.90\% which denotes the preinteraction time start possibility of failure, the post-interaction time start possibility of failure and the possible consequences of failure respectively, the output membership function strengths from the defined rules are:

$$
\begin{aligned}
& \text { "Proceed" } \left.=\sqrt{\sum(\text { proceed })}\right)^{2}=\sqrt{0.0256}=0.16 \\
& \text { "Don't Proceed" }=\sqrt{\sum(\text { Don'tproceed })}{ }^{2}=\sqrt{0.4356}=0.66
\end{aligned}
$$

Once the output membership function strength for each output has been determined, the data must be defuzzified to obtain a crisp output. The defuzzification is accomplished by combining the results of the inference process and computing the 'fuzzy centroid' of the output area. The weighted strengths of each output member function is multiplied by its respective output membership function centre points and summed. Finally, this area is divided by the sum of the weighted member function strengths and the result is taken as the crisp output. 
Determining the crisp output:

(Dont proceed_centre $*$ Dont proceed_strenght + Proceed_ce ntre $*$ Proceed_st renght)

$$
\text { (Dont proceed_st renght }+ \text { Proceed_st renght) }
$$

Substituting the respective values from above continuing example:

$$
\frac{-100 * 0.66+100 * 0.16}{0.66+0.16}=-60.97
$$

The output value of $-60.97 \%$ (60.97\%, Don't Proceed) seems logical since the particular input conditions (Pre-interaction Possibility of Failure $=3$, Post-interaction Possibility of Failure $=2$ and Consequences $=52.90 \%$ ) indicate that the possible risk associated in the interaction might be high. The minus (-) sign suggest that the output is in the 'Don't proceed' output member function.

\section{Conclusion}

It has been proven in the literature that Fuzzy logic models uncertainty and gives an output based on the vague inputs given. In this paper, we developed a methodology which incorporates a fuzzy system that assists in a risk based decision making process. The fuzzy system assists the trusting agent to decide whether to proceed or not in an interaction with a particular trusted agent based on the inputs given. The inputs given to the fuzzy system are the values related to the trusting agent's future interaction with the probable trusted agent. Based on the output from the fuzzy system, the trusting agent can either decide whether to interact or not with the trusted agent or affirm its previous decision.

\section{References}

D.F. Cooper, 'The Australian and New Zealand Standard on Risk Management, AS/NZS 4360:2004', Tutorial Notes: Broadleaf Capital International Pty Ltd. (2004) Available: http://www.broadleaf.com.au/tutorials/Tut_Standard.pdf

J. G. March and Z. Shapira, 'Managerial perspective on risk and risk taking', Management Science, vol. 33, no. 11, pp. 1404-1418.

N. Luhmann, 'Familiarity, confidence, trust: Problems and alternatives', Making and Breaking Cooperative Relations, Basil Blackwell, New York, USA.

R.C. Mayer, J.H. Davis and F.D. Schoorman, 'An interactive model for organizational trust', Academy of Management Review, vol. 20, no. 3, pp.709-734

D.M. Rousseau, S.B. Sitkin, R.S. Burt and C. Camerer, 'Not so different after all: A cross-discipline view of trust', Academy of Management Review, vol. 23, no. 3, pp. 391-404

P. Sztompka, 'Trust: A sociological theory', Cambridge University Press, Cambridge, U.K.

7. S. Grazioli and A. Wang, 'Looking without seeing: Understanding unsophisticated consumers success and failure to detect Internet deception', Proceedings of the International Conference on Information Systems, New Orleans, USA, December 16-19 200 , pp 193-204.

. C. Cheung and M.K.O. Lee, 'Trust in Internet shopping: A proposed model and measurement instrument', Proceedings of the 2000 Americas Conference on Information Systems, Long Beach, CA, August 10-13 2000, pp 681-689.

9. O.K. Hussain, E. Chang, F.K. Hussain and T.S. Dillon, 'A Methodology for Risk Based Decision Making in a Service Oriented Environment', IEEE International Conference on Sensor Networks, Ubiquitous, and Trustworthy Computing -Vol 1, (SUTC'06), pp 506-513, Taichung, Taiwan, 2006

10. J. Carter and A.A. Ghorbani, 'Towards a formalization of Trust' Web Intelligence and Agent Systems, Vol. 2, No. 3, pp. 167-183, March J. Carter

11. S. Greenland, 'Bounding analysis as an inadequately specified methodology', Risk Analysis vol. 24, no. 5, pp. 1085-1092, 2004. 